\title{
Endogenous Market Transparency and Price Competition
}

\author{
Klaus Kultti \\ University of Helsinki \\ and \\ Mikko Leppämäki \\ Helsinki School of Economics and GSF \\ and \\ Eeva Mauring \\ University of Helsinki
}

Discussion Paper No. 278

October 2009

ISSN 1795-0562

HECER - Helsinki Center of Economic Research, P.O. Box 17 (Arkadiankatu 7), FI-00014 University of Helsinki, FINLAND, Tel +358-9-191-28780, Fax +358-9-191-28781, E-mail info-hecer@helsinki.fi, Internet www.hecer.fi 


\title{
Endogenous Market Transparency and Price Competition*
}

\begin{abstract}
We introduce a model of price competition with endogenous market transparency, where it is costly for consumers to get informed about the announced prices. We show that there is symmetric mixed strategy equilibrium with a monotonic relationship between the degree of transparency and intensity of competition. Interestingly, we find that there exist multiple equilibria with zero, low and high levels of market transparency with the high level of market transparency being the stable equilibrium. Once comparing the stable equilibrium with the welfare maximizing one we find that the private market solution entails excessive investments in information acquisition. That is, at high levels of transparency the gain of consumers from increased transparency is smaller than the loss in the firms' profits leading to decrease in total welfare.
\end{abstract}

JEL Classification: D43, L13, L15

Keywords: Endogenous market transparency, price competition, mixed strategy, multiple equilibria, welfare.

Klaus Kultti and Eeva Mauring

Department of Economics, University of Helsinki P.O. Box 17 (Arkadiankatu 7) FI-00014 University of Helsinki FINLAND

e-mail: klaus.kultti@helsinki.fi

e-mail: eeva.mauring@helsinki.fi
Mikko Leppämäki

Graduate School of Finance, Helsinki School of Economics

P.O. Box 1210

FI-00101 Helsinki

FINLAND

e-mail: mikko.leppamaki@hse.fi

* We are grateful for Zhang Shilei and Natalia Nikola for research assistance. 


\section{Introduction}

It is often argued that a high market transparency is desirable for society as it fosters competition. How exactly a certain level of market transparency is achieved remains typically somewhat unclear. In this paper, we propose a model where the level of market transparency is endogenously determined. Moreover, an implication of typical argumentation, of course, is that the higher the market transparency, the higher the social welfare is. Interestingly, we conclude the opposite; in our set up the socially optimum would require lower market transparency than the private market solution entails.

We put forward a standard model of price competition, where the consumers' behaviour is not captured by the demand curve. They can also decide to acquire information on announced prices. They get to know the prevailing prices only if they acquire the price information. Our model replicates the standard Bertrand competition case when transparency, i.e., the proportion of consumers who are informed, is at the highest level. With both informed and uninformed consumers the firms' pricing is in mixed strategies. The novelty with respect to prior literature is that the degree of market transparency is endogenous as also consumers are allowed to behave strategically.

In our set up firms compete in prices, produce a homogeneous good, and have the same marginal costs of production. It is costly for consumers to become informed about prices. A consumer who acquires price information can, of course, choose the lowest price offer. The higher the proportions of informed consumers the lower the prices are in equilibrium. Thus, those consumers who do not get informed also benefit, but only on average. We show that there exists symmetric mixed strategy equilibrium in prices with a monotonic relationship between the degree of market transparency and intensity of competition.

Most interestingly, we find numerically multiple equilibria with zero, low and high levels of market transparency with the high level of market transparency being the stable equilibrium. When comparing the stable equilibrium with the social optimum, it turns out somewhat surprisingly that the social optimum would require less transparency than there is in equilibrium. That is, there is too much private investments in information acquisition. 
Of course, we are not the first ones to address market transparency. Varian (1980) is probably the pioneering study. In his model the consumers are identical, while we allow for heterogeneous valuations. There is also an old research agenda of increased market transparency leading to intensified market competition (see Stigler (1961)) as increased transparency improves the consumers' possibilities to choose goods with lowest prices. However, on the downside of increased transparency is the firms' improved possibility for collusion in a repeated relationship as increased transparency makes it easier to detect any deviations from collusive pricing.

For recent contributions, see for instance Møllgaard and Overgaard (2001) and (2002), Nilsson (1999) and Schultz (2005). While the prior literature has examined the effects of transparency on pricing, intensity of competition and welfare we fully endogenize the level of market transparency by introducing the consumers' strategic behavior in an otherwise standard model of price competition. Moreover, our finding of multiple equilibria of market transparency is new in the literature.

In search theoretic framework Lester (2009) finds that while firms are capacityconstrained the relationship between transparency and prices is not as clear as prior theoretical literature has argued. In particular, higher transparency can lead to either higher or lower prices. Schultz (2004) analyzes the effects of market transparency in a Hotelling model where a fraction of consumers are informed about the prices and locations of firms. He shows that increased transparency leads to unambiguous improvement in consumers' and total welfare. Our welfare results with respect to transparency and consumers' welfare are in line with those of Schultz (2004) while we demonstrate that the effect of increased transparency on total welfare is ambiguous. Namely, at high levels of transparency the gain of consumers from increased transparency is smaller than the loss in the firms' profits leading to decrease in total welfare. 


\section{The Basic Setup}

Consider a mass of consumers normalized to unity. Assume that their valuations of a homogenous commodity are independent draws from the uniform distribution on unit interval. Once the valuations are realized there is a linear demand curve $q=1-p$. Notice that other distributions would, of course, generate different demand curves. Assume further that there are two firms which produce a homogenous good with constant marginal cost normalized to zero. Let us call these firms $A 1$ and $A 2$. Below we denote the proportion of those consumers who have observed the firms' announcements by $\theta$, and this is intended to be a measure of market transparency. That is, proportion $1-\theta$ of consumers have observed nothing, and will visit the firms randomly.

To construct an equilibrium we assume that consumers use a symmetric mixed strategy. The firms' behaviour is then necessarily symmetric.

Definition 1 An equilibrium is a proportion $\theta \in[0,1]$ of observing consumers and prices $p_{1}$ and $p_{2}$ s.t. no consumer wants to deviate and the prices maximize the firms' profits.

\section{Price Competition: Symmetric Equilibrium}

Consider the price competition where the firms $A 1$ and $A 2$ announce their prices $p_{1}$ and $p_{2}$ simultaneously, and assume further that proportion $\theta$ of consumers observe the announced prices. In a symmetric equilibrium both firms then get $\frac{1-\theta}{2}$ consumers to start with, and they compete for the $\theta$ consumers. If the firms compete in prices in a Bertrand-fashion it is clear that for $\theta>0$ there does not exist a symmetric pure strategy equilibrium for exactly the same reason as in the standard Bertrand-competition case.

There, however, exists a symmetric equilibrium in mixed strategies and that is what we construct next. The support of the mixed strategy $F$ is a closed interval $[\underline{p}, \bar{p}]$ and it has no mass points (see Kultti and Virrankoski, 2003 for the reasoning and proof in an analogous case). It is clear that the highest price is never higher than the monopoly price. Also, it cannot be lower since when 
a firm chooses the highest price it only gets the non-observing consumers and might as well charge them the monopoly price.

Assume that $A 2$ uses $F$ and that $A 1$ chooses price $p$ from the support of the mixed strategy. $A 1$ 's profit is then

$$
F(p) \frac{1-\theta}{2}(1-p) p+(1-F(p))\left(\frac{1-\theta}{2}+\theta\right)(1-p) p .
$$

This magnitude must be constant for all $p$ in the support of the mixed strategy. In particular, it must equal the profit at the monopoly price $p^{m}=\frac{1}{2}$. Notice that at this price $F\left(p^{m}\right)=1$. Equating (1) and the profit of $A 1$ at the monopoly price yields the following formula for the mixed strategy

$$
F(p)=\frac{4(1+\theta)(1-p) p-(1-\theta)}{8 \theta(1-p) p} .
$$

To determine $\underline{p}$ notice that $F(\underline{p})=0$. Now equating the monopoly profit and the profit at $\underline{p}$ yields the following condition

$$
\underline{p}^{2}-\underline{p}+\frac{1-\theta}{4(1+\theta)}=0
$$

The sensible root is

$$
\underline{p}=\frac{1-\sqrt{\frac{2 \theta}{1+\theta}}}{2}
$$

as the other root is greater than the monopoly price. To summarize we have the following result:

Proposition 2 There exists an equilibrium where prices are on the interval $[\underline{p}, \bar{p}]$, and where $\underline{p}=\frac{1-\sqrt{\frac{2 \theta}{1+\theta}}}{2}$ and $\bar{p}=\frac{1}{2}$.

That is, when $\theta=1$ the lower limit $\underline{p}$ is at the competitive level with two firms, and at $\theta=0$ the lower limit $\underline{p}$ is at the monopoly level of a single firm. Thus, we have a nice and smooth transition from the monopoly price to the competitive price in a duopoly when the proportion of the informed consumers goes from zero to unity. The intensity of market competition is thus increasing in market transparency and quite naturally consumers are then also better off due to the lower prices. Let us next examine consumers' incentives to become informed about announced prices in the first place. 


\section{Transparency in Equilibrium}

Assume that consumers have to pay a fixed cost of $\gamma$ to become informed about the firms' posted prices. We want to find the equilibrium proportion of consumers who decide to become informed. In equilibrium the consumers must be indifferent between observing the prices and not observing them.

First we determine the expected utility of a consumer who does not incur the cost of becoming informed. He chooses one of the firms at random and the expression for his utility is

$$
\int_{\underline{p}}^{p^{m}} \int_{\underline{p}}^{v}(v-p) f(p) d p d v+\int_{p^{m}}^{1} \int_{\underline{p}}^{p^{m}}(v-p) f(p) d p d v .
$$

The above expression gives the consumer's expected utility when the firm has set price $p$ which happens with probability $f(p)$. In (5) the first part is the expected utility of the consumer whose valuation $v$ is on the interval $\left[\underline{p}, p^{m}\right]$, and when the price $p$ set by the firm lies in the interval $[\underline{p}, v]$. Notice that in the consumer's valuation the lower limit of integral starts from $\underline{p}$ instead of zero, since all those consumers whose valuation is lower than $\underline{p}$ will not buy the good and thus receive zero. The second part of (5) gives the consumer's expected utility when his valuation is at least the monopoly price. Thus, in the first integral the upper limit hits the maximum valuation of $v=1$.

After some routine algebra (executed in Appendix 1) expression (5) can be developed into form

$$
=\frac{1-\theta}{16 \theta}\left[\frac{2}{1-\sqrt{\frac{2 \theta}{1+\theta}}}-2+2 \ln \left(1-\sqrt{\frac{2 \theta}{1+\theta}}\right)\right] .
$$

Consider next the case when consumers can learn the announced prices. By paying $\gamma$ a consumer observes the prices announced by $A 1$ and $A 2$, and then gets to choose the lower price, i.e., he gets to choose the lower of two identically

distributed random variables. The probability $g(p)$ that the lower of the two prices is $p$ is

$$
g(p)=2 f(p)(1-F(p))=\frac{(1-\theta)(1-2 p)}{4 \theta(1-p)^{2} p^{2}}\left[\frac{(1-\theta)}{8 \theta(1-p) p}-\frac{1-\theta}{2 \theta}\right] .
$$


Now the expected utility of a consumer who observes the prices can be expressed as follows

$$
\int_{\underline{p}}^{p^{m}} \int_{\underline{p}}^{v}(v-p) g(p) d p d v+\int_{p^{m}}^{1} \int_{\underline{p}}^{p^{m}}(v-p) g(p) d p d v-\gamma .
$$

By paying $\gamma$ a consumer becomes informed about the prices and chooses the lowest one, i.e., price $p$. Otherwise the expression of the expected utility remains the same as in (5). After some rather involved but routine algebra (see Appendix 2) equation (8) turns out to equal

$$
\frac{(1-\theta)^{2}}{16 \theta^{2}}\left[\frac{4\left(\sqrt{\frac{2 \theta}{1+\theta}}\right)^{2}-3 \sqrt{\frac{2 \theta}{1+\theta}}}{2\left(1-\sqrt{\frac{2 \theta}{1+\theta}}\right)^{2}}-2 \ln \left(1-\sqrt{\frac{2 \theta}{1+\theta}}\right)+\frac{1}{4} \ln \frac{1-\sqrt{\frac{2 \theta}{1+\theta}}}{1+\sqrt{\frac{2 \theta}{1+\theta}}}\right]-\gamma .
$$

In equilibrium a consumer must be indifferent between observing and not observing the prices which condition determines the equilibrium value of $\theta$. That is, by equating (6) and (9) one should in principle be able to solve $\theta$. It should not come as a surprise that it is impossible to solve such $\theta$ in a closed form. We solve the model numerically, and present graphics for the expected utilities.

Assuming that the cost of getting informed, $\gamma$, takes values in the set $\{0,0.02,0.05\}$ we can plot (6) and (9) in the same picture. 


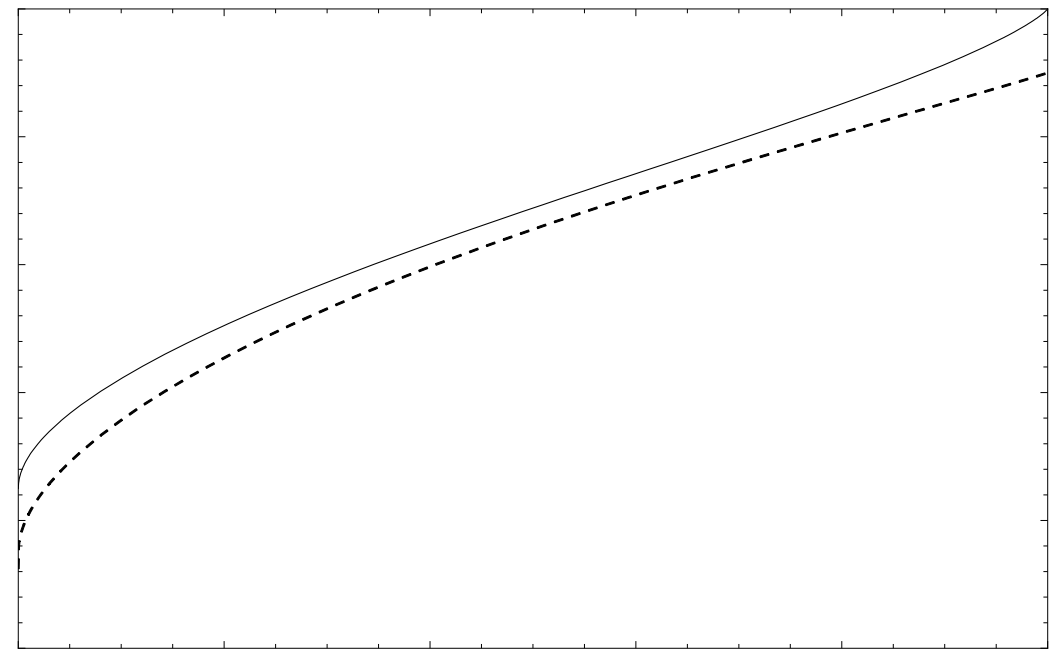

Figure 1: Observer's (dashed) and unobserver's (solid) expected utilities at $\gamma$ $=0.05$. 


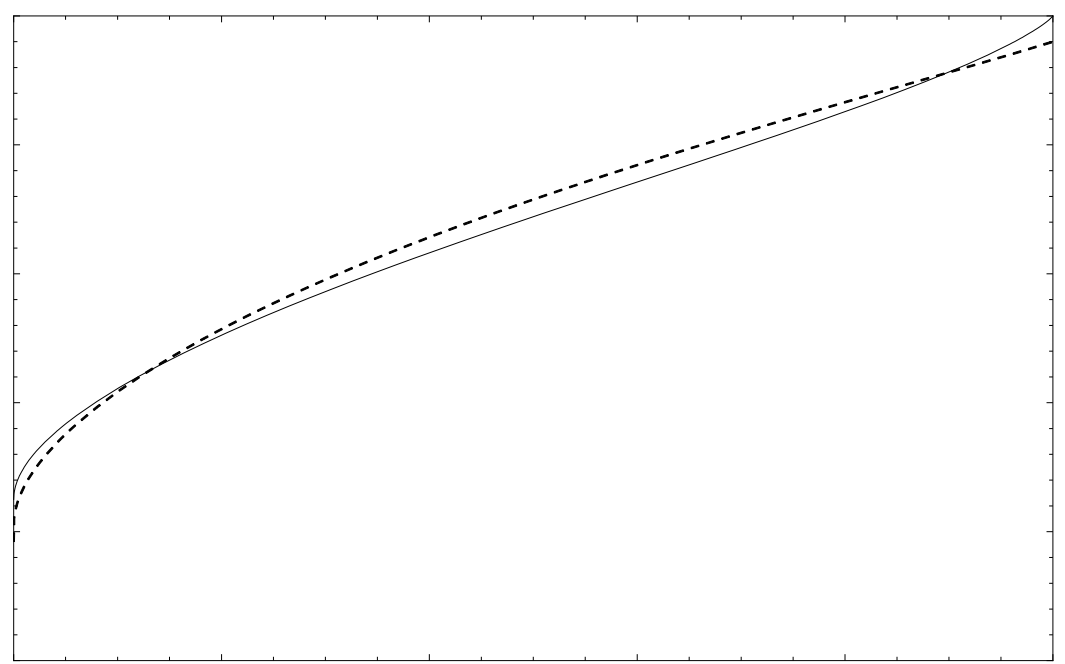

Figure 2: Observer's (dashed) and unobserver's (solid) expected utilities at $\gamma$ $=0.02$.

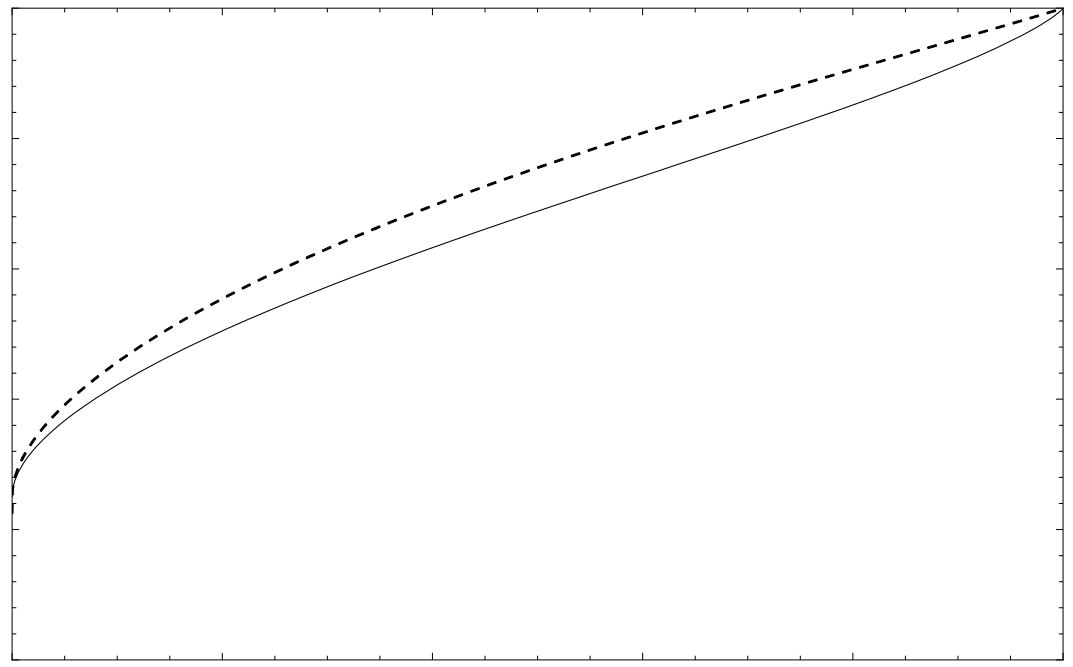

Figure 3: Observer's (dashed) and unobserver's (solid) expected utilities at $\gamma$ $=0$. 
We can summarize the results with different levels of cost of getting informed, $\gamma$, as follows:

Proposition 3 There is always an equilibrium where no-one observes. When $\gamma>\bar{\gamma}$ there is a unique equilibrium where no-one observes. When $\gamma \in(0, \bar{\gamma}]$ there are multiple (three) equilibria with zero, low and high levels of market transparency i.e. where $\theta \in[0,1)$. If $\gamma=0$ there exists two equilibria with either $\theta=0$ or $\theta=1$.

Somewhat surprisingly it turns out that there are multiple equilibria. On reflection this is easy to understand; what matters to a consumer is the marginal utility from observing the prices. When very few consumers observe the price it does not pay to observe the price since the support of the firms' mixed strategy is not very large, and thus getting the lower of the two prices results in not-so-big reduction in the price. When many consumers observe the price the support of the mixed strategy is large and getting the lower price results in a big reduction in the price. Of the two equilibria where $\theta>0$, the one of higher transparency is the stable equilibrium. Notice that there is also a third equilibrium where no one invests in getting informed, and then $\theta=0$. Quite naturally when the cost of getting informed $\gamma$ increases it does not pay to become informed any longer, and thus the curve representing the expected utility of an observing consumer moves downwards.

\section{Welfare}

To determine how the market equilibrium fares compared to the social optimum we postulate that the social welfare measure is the sum of profits and consumer surplus. This is easy to calculate as proportion $\theta$ of consumers gets the expected utility in expression (9) and proportion $1-\theta$ gets the expected utility in expression (6). There are also two firms both of which make the same expected profit

which is the same as the profit at the monopoly price (since it belongs to the support of the mixed strategy). Formally, the social welfare measure is 


$$
\begin{aligned}
& \theta\left[\frac{(1-\theta)^{2}}{16 \theta^{2}}\left(\frac{4\left(\sqrt{\frac{2 \theta}{1+\theta}}\right)^{2}-3 \sqrt{\frac{2 \theta}{1+\theta}}}{2\left(1-\sqrt{\frac{2 \theta}{1+\theta}}\right)^{2}}-2 \ln \left(1-\sqrt{\frac{2 \theta}{1+\theta}}\right)+\frac{1}{4} \ln \frac{1-\sqrt{\frac{2 \theta}{1+\theta}}}{1+\sqrt{\frac{2 \theta}{1+\theta}}}\right)-\gamma\right]+ \\
& +(1-\theta)\left[\frac{1-\theta}{16 \theta}\left(\frac{2}{1-\sqrt{\frac{2 \theta}{1+\theta}}}-2+2 \ln \left(1-\sqrt{\frac{2 \theta}{1+\theta}}\right)\right)\right]+\frac{1}{4}(1-\theta) .
\end{aligned}
$$

Which can be consolidated into the following form

$$
\frac{(1-\theta)^{2}}{16 \theta}\left[\frac{\sqrt{\frac{2 \theta}{1+\theta}}}{2\left(1-\sqrt{\frac{2 \theta}{1+\theta}}\right)^{2}}+\frac{1}{4} \ln \frac{1-\sqrt{\frac{2 \theta}{1+\theta}}}{1+\sqrt{\frac{2 \theta}{1+\theta}}}\right]-\theta \gamma+\frac{1}{4}(1-\theta) .
$$

The graph of the social welfare measure (11) shows that welfare is a concave function, increasing in transparency $\theta$ till a unique maximum is reached and decreasing thereafter (the latter with positive cost of getting informed) as seen in figure 4. Increase in the cost of obtaining information $\gamma$ shifts the tail of the curve downwards, here plotted at values $0,0.01,0.02$ and 0.03 .

Taking the first order condition of the welfare function with respect to $\theta$ yields the following condition for the social optimum

$\frac{1-\theta}{16 \theta}\left[\frac{2}{\left(1-\sqrt{\frac{2 \theta}{1+\theta}}\right)^{2}(1+\theta)}-\frac{\sqrt{\frac{2 \theta}{1+\theta}}(1+\theta)}{2 \theta\left(1-\sqrt{\frac{2 \theta}{1+\theta}}\right)^{2}}-\frac{1+\theta}{4 \theta} \ln \frac{1-\sqrt{\frac{2 \theta}{1+\theta}}}{1+\sqrt{\frac{2 \theta}{1+\theta}}}\right]-\gamma-\frac{1}{4}=0$

It is of some interest to compare the privately chosen stable equilibrium with high level of market transparency $\theta_{p}$ with that of the welfare maximizing $\theta_{s}$ chosen by the social planner. Since from (12) it is impossible to solve $\theta$ in a closed form we use instead numerical analysis and solve both the private and social optimum and compare those.

Assuming the cost of getting informed will take values $0,0.01,0.02$ and 0.03 , we can solve the private equilibrium value of transparency, $\theta_{p}$ by equating (6) 


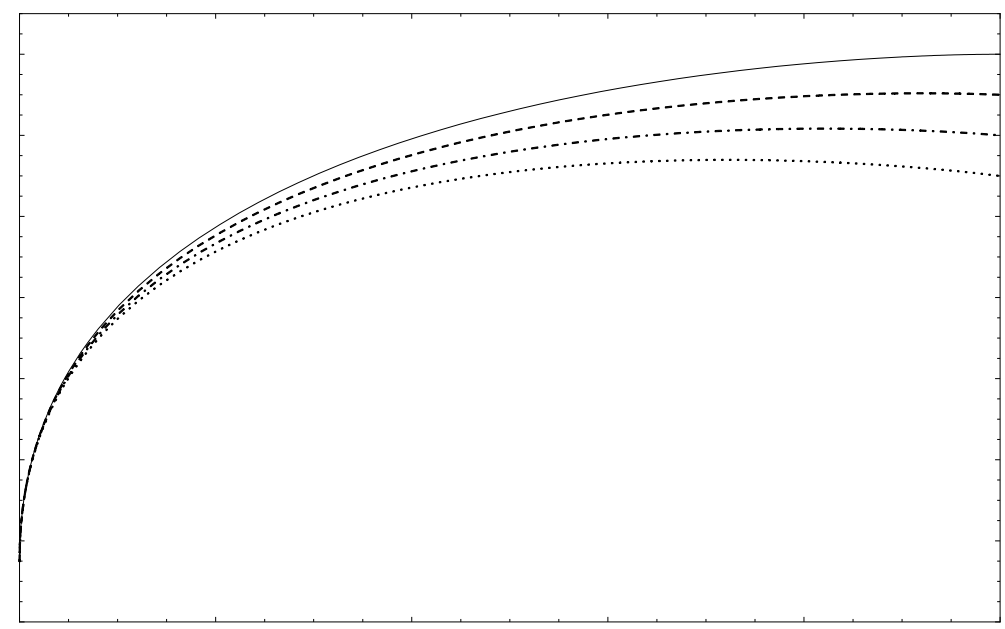

Figure 4: Total utilities at $\gamma$ values 0 (solid), 0.01 (dashed), 0.02 (dot-dashed), 0.03 (dotted).

and (9). Similarly by plugging in (12) different values of $\gamma$ we can derive the socially optimal level of transparency, $\theta_{s}$. These values are reported below in table 1.

Table 1: Comparison of privately chosen equilibrium $\theta_{p}$ and welfare maximizing $\theta_{s}$

$$
\begin{aligned}
& \gamma=0 \quad \theta_{p}=1 \quad \theta_{s}=1 \\
& \gamma=0.01 \quad \theta_{p}=0.97 \quad \theta_{s}=0.92 \\
& \gamma=0.02 \quad \theta_{p}=0.90 \quad \theta_{s}=0.82 \\
& \gamma=0.03 \quad \theta_{p}=0.74 \quad \theta_{s}=0.72
\end{aligned}
$$

We find that for all positive values of $\gamma$ (up to $\bar{\gamma}$ after which no one observes) there is too much market transparency in the optimal private market solution compared to social optimum. The intuition behind this result is that for low levels of transparency the gain of consumers from increased transparency exceeds the loss in the firms' profits, as shown in figure 5. However, at higher 


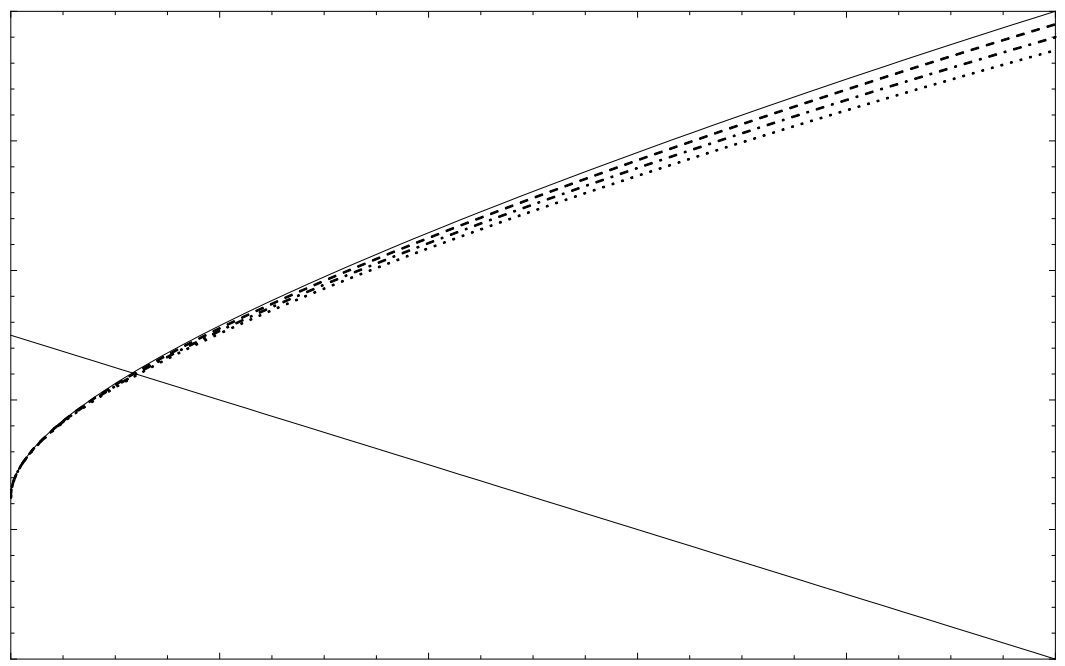

Figure 5: Growing consumers' surplus at $\gamma\{0,0.01,0.02,0.03\}$ and declining profits.

values of transparency the loss in the firms' profits outweighs the increase in consumers' welfare. The figure shows that if at low levels of $\theta$ the slope of the curves representing the consumers' welfare is bigger in absolute terms than of firms' profits, then the situation is the opposite at higher values of transparency. Thus, if the social planner assigns equal weight to the well-being of both firms and consumers, then socially optimal level of market transparency is lower than that of privately optimal. Note also that if firms' profits decreased non-linearly in transparency, then the result would change depending on the relative decrease of the firms' utility compared to that of consumers.

Proposition 4 The private and socially optimal levels of market transparency coincide only when the cost of getting informed is zero. For all other values of $\gamma$ the optimal private market solution involves too much transparency. 


\section{Conclusion}

We have developed a model of endogenous transparency where it is costly for consumers to get informed about the prices announced by the firms. We show that there is a symmetric mixed strategy equilibrium that we derive explicitly. We demonstrate that there is a monotonic relationship between the degree of transparency and intensity of competition; the prices and profits decrease from monopoly level to competitive level as transparency increases from zero to perfect transparency. It is clear that there always exists an equilibrium, if nothing else then an equilibrium where no-one acquires information. Numerical analysis reveal that there exist multiple equilibria (exactly three) with zero, low and high levels of market transparency. Zero and high levels of transparency are stable equilibria whereas the low level is unstable.

Quite surprisingly, it turns out that the stable equilibrium with strictly positive transparency entails higher than socially optimal level of information acquisition. The market solution features too much transparency. This finding is contrary to the results in the papers mentioned in the introduction. It also runs counter to the often mentioned requirements to increase transparency in various markets, the financial markets being the leading example.

There are two reasons for too high transparency in equilibrium. One is that the agents' decision to acquire information makes price competition more fierce, and lowers the profits of the firms. As profits are part of the welfare it may well go down. The other reason emanates from the fact that the analysis is of partial equilibrium type. In a general equilibrium model the consumers would also be the owners of the firms and the firms' profits would be distributed to the consumers. Taking into account these general equilibrium effects might well change the result.

\section{References}

[1] Kultti, K. and J. Virrankoski 2003: Price Distribution in a Symmetric Economy.Topics in Macroeconomics 3. http://www.bepress.com/bejm/topics/vol3/iss1/art5 
[2] Lester, B. 2009: Information and Prices with Capacity Constraints: mimeo. University of Western Ontario.

[3] Møllgaard, H.P. and P.B. Overgaard 2001: Market Transparency and Competition Policy. Rivista di Politica Economica 91. 11-58.

[4] Møllgaard, H.P. and P.B. Overgaard 2002: Market Transparency: A Mixed Blessing? University of Copenhagen. mimeo.

[5] Nilsson, A. 1999: Transparency and Competition. Stockholm School of Economics. Working Paper Series in Economics and Finance No. 298.

[6] Schultz, C. 2004: Market Transparency and Product Differentiation. Economics Letters 83. 173 - 178.

[7] Schultz, C. 2005: Transparency on the Consumer Side and Tacit Collusion. European Economic Review 49. 279-297.

[8] Stigler, G.J. 1961: The Economics of Information. The Journal of Political Economy 69. 213-225.

[9] Varian, H. 1980: A Model of Sales. American Economic Review 70. 651 659. 


\section{Appendix 1: Derivation of expected utility of unin- formed consumer.}

$$
\int_{\underline{p}}^{p^{m}} \int_{\underline{p}}^{v}(v-p) f(p) d p d v+\int_{p^{m}}^{1} \int_{\underline{p}}^{p^{m}}(v-p) f(p) d p d v
$$

It is useful to change the order or integration as follows

$$
\begin{gathered}
=\int_{\underline{p}}^{p^{m}} \int_{p}^{p^{m}}(v-p) d v f(p) d p+\int_{\underline{p}}^{p^{m}} \int_{p^{m}}^{1}(v-p) d v f(p) d p \\
=\int_{\underline{p}}^{p^{m}}\left[/_{p}^{p^{m}}\left(\frac{1}{2} v^{2}-p v\right)+\int_{p^{m}}^{1}\left(\frac{1}{2} v^{2}-p v\right)\right] f(p) d p \\
=\int_{\underline{p}}^{p^{m}}\left[\frac{1}{2} p^{m^{2}}-\frac{1}{2} p^{2}-p p^{m}+p^{2}+\frac{1}{2}-\frac{1}{2} p^{m^{2}}-p+p p^{m}\right] f(p) d p \\
=\frac{1}{2} \int_{\underline{p}}^{p^{m}}(1-p)^{2} f(p) d p .
\end{gathered}
$$

Now we can substitute in $f(p)=F^{\prime}(p)$

$$
\begin{gathered}
=\frac{1}{2} \int_{\underline{p}}^{p^{m}}(1-p)^{2} \frac{(1-\theta)(1-2 p)}{8 \theta(1-p)^{2} p^{2}} d p . \\
=\frac{1}{2} \frac{(1-\theta)}{8 \theta} \int_{\underline{p}}^{p^{m}} \frac{(1-2 p)}{p^{2}} d p \\
=\frac{(1-\theta)}{16 \theta} \int_{\underline{p}}^{p^{m}}\left(\frac{1}{p^{2}}-\frac{2}{p}\right) d p \\
=\frac{(1-\theta)}{16 \theta} /_{\underline{p}^{m}}^{p^{m}}\left(-\frac{1}{p}-2 \ln p\right)
\end{gathered}
$$

after substituting $p^{m}=\frac{1}{2}$ and $\underline{p}=\frac{1-\sqrt{\frac{2 \theta}{1+\theta}}}{2}$ we have 


$$
=\frac{(1-\theta)}{16 \theta}\left[\frac{2}{1-\sqrt{\frac{2 \theta}{1+\theta}}}-2+2 \ln \left(1-\sqrt{\frac{2 \theta}{1+\theta}}\right)\right]
$$

\section{Appendix 2: Derivation of expected utility of informed consumer}

$$
\int_{\underline{p}}^{p^{m}} \int_{\underline{p}}^{v}(v-p) g(p) d p d v+\int_{p^{m}}^{1} \int_{\underline{p}}^{p^{m}}(v-p) g(p) d p d v-\gamma
$$

Again it is useful to change the order or integration as follows

$$
\int_{\underline{p}}^{p^{m}} \int_{p}^{p^{m}}(v-p) d v g(p) d p+\int_{\underline{p}}^{p^{m}} \int_{p^{m}}^{1}(v-p) d v g(p) d p-\gamma
$$

We forget $\gamma$ for a moment and develop the the first two expressions. As in the case of the uniformed consumer we get the following expression

$$
=\frac{1}{2} \int_{\underline{\underline{p}}}^{p^{m}}(1-p)^{2} g(p) d p
$$

recalling that $g(p)=2 f(p)(1-F(p))$ we get

$$
\begin{gathered}
\frac{1}{2} \int_{\underline{p}}^{p^{m}}(1-p)^{2} \frac{(1-\theta)(1-2 p)}{4 \theta(1-p)^{2} p^{2}}\left[\frac{(1-\theta)}{8 \theta(1-p) p}-\frac{1-\theta}{2 \theta}\right] d p \\
\quad=\frac{1}{2} \frac{(1-\theta)^{2}}{8 \theta^{2}} \int_{\underline{p}}^{p^{m}} \frac{(1-2 p)}{p^{2}}\left[\frac{1}{4(1-p) p}-1\right] d p
\end{gathered}
$$

Now we can develop

$$
\int_{\underline{p}}^{p^{m}} \frac{(1-2 p)}{p^{2}}\left[\frac{1}{4(1-p) p}-1\right] d p
$$


further on in the following form

$$
\begin{gathered}
\int_{\underline{p}}^{p^{m}}\left[\frac{(1-p)}{p^{2}}\left(\frac{1}{4 p(1-p)}-1\right)\right] d p-\int_{\underline{p}}^{p^{m}} \frac{p}{p^{2}}\left(\frac{1}{4 p(1-p)}-1\right) d p \\
=\int_{\underline{p}}^{p^{m}} \frac{1}{4 p^{3}} d p-\int_{\underline{p}}^{p^{m}} \frac{1-p}{p^{2}} d p-\int_{\underline{p}}^{p^{m}} \frac{1}{4 p^{2}(1-p)} d p+\int_{\underline{p}}^{p^{m}} \frac{1}{p} d p \\
=\left[-\frac{1}{8 p^{2}}+\frac{1}{p}+\ln p+\frac{1}{4 p}-\frac{1}{4} \ln p+\frac{1}{4} \ln (p-1)+\ln p\right]_{\underline{p}}^{p^{m}}
\end{gathered}
$$

Plug in above

$p^{m}=\frac{1}{2}$

$\underline{p}=\frac{1-\sqrt{\frac{2 \theta}{1+\theta}}}{2}$

and then solve the expression, and one gets the following expression for the payer's

expected utility where the multiplier $\frac{(1-\theta)^{2}}{16 \theta^{2}}$ and the cost of getting informed, $\gamma$ are reintroduced

$$
\frac{(1-\theta)^{2}}{16 \theta^{2}}\left[\frac{4\left(\sqrt{\frac{2 \theta}{1+\theta}}\right)^{2}-3 \sqrt{\frac{2 \theta}{1+\theta}}}{2\left(1-\sqrt{\frac{2 \theta}{1+\theta}}\right)^{2}}-2 \ln \left(1-\sqrt{\frac{2 \theta}{1+\theta}}\right)+\frac{1}{4} \ln \frac{1-\sqrt{\frac{2 \theta}{1+\theta}}}{1+\sqrt{\frac{2 \theta}{1+\theta}}}\right]-\gamma
$$

